\title{
Interplay of electric field and disorder in Dirac liquid silicene
}

\author{
L. Craco ${ }^{1,2, a}$, S. S. Carara ${ }^{1}$, and S. Leoni ${ }^{3}$ \\ 1 Institute of Physics, Federal University of Mato Grosso, Cuiabá, MT 78060-900, Brazil \\ 2 Leibniz Institute for Solid State and Materials Research Dresden, 01069 Dresden, Germany \\ 3 School of Chemistry, Cardiff University, Cardiff CF10 3AT, UK
}

Received 6 October 2020 / Accepted 15 January 2021 / Published online 8 February 2021 (C) The Author(s) 2021

\begin{abstract}
.
Abstract Layered materials with buckled structure offer a promising route to explore distinct phases of quantum matter. Using GGA + DMFT we reveal the complex interplay between perpendicular electric field and site-diagonal disorder in the Dirac liquid electronic state of silicene. The electronic structure we derive is promising in the sense that it leads to results that might explain why out-of-plane electric field plus moderate disorder can generate marginal Dirac valleys consistent with scanning tunneling spectroscopy of silicene on Ag substrates.
\end{abstract}

\section{Introduction}

Silicene, the silicon-based counterpart of two-dimensional (2D) graphene, has attracted much attention both experimental and theoretically. [1-14]. Likewise graphene, silicene displays Dirac cones at $K$ points near the Fermi level $\left(E_{F}\right)$ arising from the nearly linear band dispersion of $3 p_{z}$ ( $\pi$-symmetry) [1,4] electronic states. The similarity between silicene and graphene arises from the fact that $\mathrm{Si}$ and $\mathrm{C}$ belong in the same column IV of the periodic table of elements. However, this close relative of graphene have a larger ionic radius which promotes $s p^{3}$ hybridization in contrast to graphene where the $s p^{2}$ hybridization is energetically favorable. As a result, in the $2 \mathrm{D}$ honeycomb lattice of $\mathrm{Si}$ atoms, the bonding is formed by mixed $s p^{2}$ and $s p^{3}$ hybridizations $[4,11]$. Hence, the most important structural difference between silicene and graphene lies in that, while the graphene layer forms a regular flat plane, the silicene layer instead takes the form of noncoplanar low-buckled structure, with one of the two sublattices of the honeycomb lattice being displaced vertically with respect to the other, as displayed in Fig. 1. This slightly buckled lattice structure is important for tuning the electronic structure of silicene using perpendicular electric fields and, thus, for opening an electrically tunable band gap $[4,9]$ as well as for electric field induced superconducting and localized magnetic states silicene $[15,16]$.

Breaking the sublattice symmetry of the honeycomb lattice leads to massive Dirac fermions [17], which similar to graphene can be enhanced via combined strain and Coulomb correlation effects [18]. Therefore, sil- icene is a promising candidate to study both massless and massive Dirac fermions under external perturbations like strain and electric field $[19,20]$. It is, however, expected that by combining many-body electronelectron interactions [10] with out-of-plane electric fields $[15,16,21]$ and local disorder [22-24], a wider range of tunability in the electronic structure of silicene might exist. How will these three (intrinsic or not) factors affect the electronic structure of silicene? To address this question we carried out the correlated band structure calculations [25] to reveal the electronic structure reconstruction of silicene. However, before delving into this problem of fundamental and applied importance, it is worth mentioning that due to larger lattice constant of silicene as compared to graphene, the hopping amplitude $t$ between the neighboring atoms in silicene is smaller as compared to graphene [10]. While the Hubbard $U$ in graphene is near to $10.0 \mathrm{eV}$, the estimate on-site Coulomb repulsion in silicene is $4.77 \mathrm{eV}$ [10], i.e., close to $5.0 \mathrm{eV}$.

As a side remark, it has been recognized in recent years the importance of understanding the correlated electronic state of honeycomb lattice systems [26], including silicene $[10,15,16]$, bilayer silicene [27], graphene $[18,28,29]$, graphite [30] and Bi-based Dirac fermion systems [31-33]. With this in place, in this work we perform realistic density functional plus dynamical mean-field theory (DFT + DMFT) calculations [25] to explore the role played by intrinsic multi-orbital (MO) electron-electron interactions in silicene and the interplay of electric field and site-diagonal disorder, revealing a marginal Dirac liquid regime in disordered silicene with characteristics akin to quasi-freestanding

\footnotetext{
${ }^{\mathrm{a}}$ e-mail: lcraco@fisica.ufmt.br (corresponding author)
} 


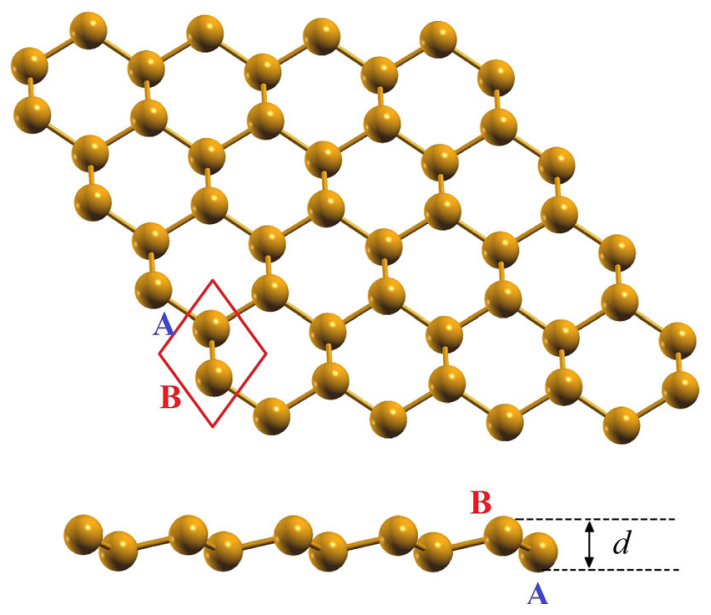

Fig. 1 Side (a) and planar (b) view of the noncoplanar low-buckled lattice structure of silicene, with sublattices $A$ and $B$ forming two separated plains. In the lower panel, $d$ is the sublattice buckling distance

graphene [28] and unconventional superconductors [34, $35]$.

The underlying motivation to our proposal comes from experimentally observed deviations from a Dirac liquid behavior in scanning tunneling spectroscopy (STS) data [3,11], suggesting the possible interplay between electronic correlations, electric field and multiphase or substract-induced disorder effects in silicene [8]. Hence, we opine that a treatment which describes dynamical electronic correlations plus disorder [24] as well as electric field effects [21] on an equal footing is of fundamental importance to Dirac fermion semimetals, where charge carriers interact via Coulomb interaction $[15,16]$. However, before delving into this problem we shall notice here an earlier DMFT study [26], discussing the notion of Dirac liquid as a fixed point of interacting Dirac fermions. Consistent with our results below, Ref. [26] has shown that an interacting Dirac sea is essentially an effective free Dirac theory. This, in turn, implies that the Dirac linear spectra at low energies remains stable against many-body Coulomb interactions [36]. The sole role of interactions in the weakly correlated Dirac liquid regime is to renormalize the Dirac quasiparticles, promoting a gradual reduction of the Dirac-band edges in the correlated spectra $[18,26]$.

In earlier studies we have shown that the frequencydependence of self-energy imaginary parts in the clean limit of the Dirac liquid electronic state vanishes at low energies $[18,37]$, implaying that interacting Dirac liquid semimetals are correlated electron systems whose excitations and normal state properties are adiabatically connected to noninteracting Dirac fermions near the linear relativistic band dispersion. This behavior is, on the other hand, in contrast with that found in marginal Fermi liquids, where the self-energy imaginary part is linear in frequency. For the sake of clarity, we recall here Varma et al. [38] work, where a marginal Fermi liquid theory for the cuprate oxide supercon- ductors was introduced. Aiming for a phenomenological explanation of strong deviations from a conventional Fermi liquid metal, Varma et al. proposed a momentum independent self-energy arising from charge and spin fluctuations of the form $\Sigma_{m F L}(\omega, T) \sim$ $g^{2} \rho^{2}(0)\left[\omega \ln \frac{x}{\omega_{c}}-i \frac{\pi}{2} x\right]$, where $g$ is a coupling constant, $\rho(0)$ the bare one-particle density-of-states (DOS) at zero frequency, $x=\max (|\omega|, T)$, and $\omega_{c}$ is an ultraviolet cutoff. This phenomenological approach assumes that the energy-dependent local self-energy behaves like $\operatorname{Re} \Sigma_{m F L}(\omega) \sim \omega \ln |\omega|$ and $\operatorname{Im} \Sigma_{m F L}(\omega) \sim|\omega|$ in contrast to normal Fermi liquid metals where $\operatorname{Re} \Sigma_{F L}(\omega) \sim$ $-\omega$ and $\operatorname{Im} \Sigma_{F L}(\omega) \sim-\omega^{2}$ holds true at low energies [39]. Interestingly, an early random phase approximation (RPA) study [40] has shown that Dirac liquid might host similar strange marginal Fermi liquid behavior with an imaginary part that is linear in frequency, meaning that under proper self-energy corrections marginal Fermi liquidness can also emerge in interacting Dirac liquid semimetals. With these caveats in mind, here we derive a quantum critical mechanism for the emergence of an orbital-selective marginal Dirac liquid electronic state in disordered silicene.

\section{Theory and results}

\subsection{GGA results}

Our first-principles calculations were performed using DFT as implemented in the SIESTA simulation package. [41,42] The respective Brillouin zone for geometry optimization and convergence of the total energy is sampled by a $20 \times 20 \times 1$ Monkhorst-Pack grid [43] The $k$-points grid used to evaluate the freestanding silicene DOS in our work is sampled by a $60 \times 60 \times 1$ Monkhorst-Pack grid. Norm-conserving pseudopotentials of Troullier-Martins [44] in Kleinman-Bylander nonlocal form were used to represent the ionic core potential. We use the generalized gradient approximation (GGA), as parameterized by Perdew et al. scheme [45], for the exchange-correlation functional. The Kohn-Sham orbitals [46] are expanded in a linear combination of atomic orbitals of the finite range which is determined by a common confinement energy shift of 0.01 Ry. [47] The precision of the real-space grid integration is determined by a minimal energy cutoff of $200 \mathrm{Ry}$ [48]. To ensure that there is no interaction between successive periodic images a vacuum region of $30 \AA$ was introduced to eliminate any artificial interaction between neighboring supercells along the perpendicular $z$ direction. Finally, to be consistent with earlier band structure studies, the lattice constant [4] and the buckled separation [20] used in our GGA calculation for pristine silicene are, respectively, $a_{0}=3.86 \AA$ and $\Delta_{0}=0.46 \AA$ [2]. With this, in Fig. 2 we display the band structure of freestanding silicene at zero and in the presence of out-of-plane electric field. As expected, at zero field it resembles the band structure of graphene, 


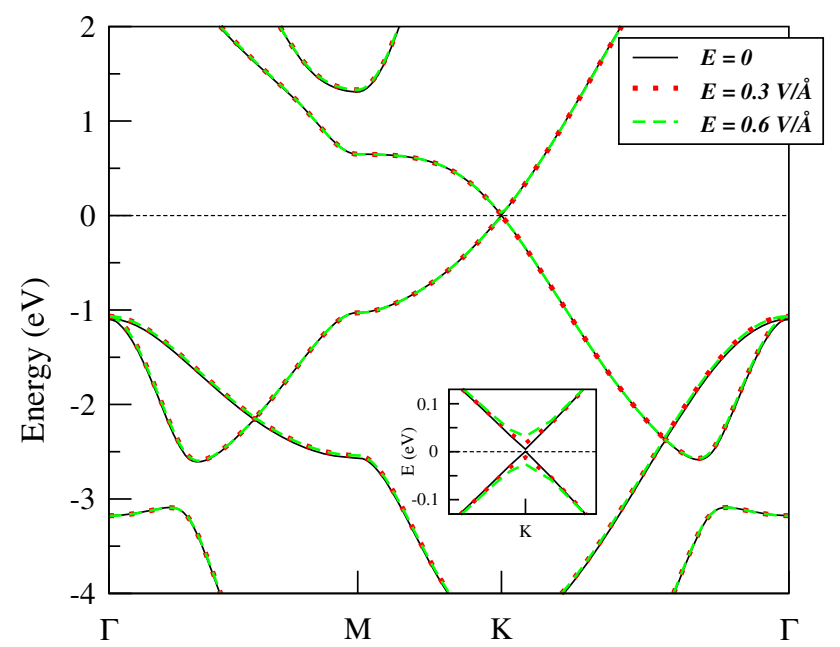

Fig. 2 GGA band structure for silicene in zero and finite perpendicular external electric fields $(E)$. Inset shows the spectrum near the Fermi level in the vicinity of the $\mathrm{K}$ point

in particular, it shows the linear Dirac-type dispersion of electrons near the $\mathrm{K}$ point. Moreover, consistent with earlier studies $[4,11,20]$, the main effect of the perpendicular electric field (see inset of Fig. 2) is to open an energy gap at the $\mathrm{K}$ point of the hexagonal Brillouin zone due to break of the symmetry between the $A$ and B sublattices of the honeycomb lattice structure. However, the screening of the electric potential by the sublattice polarization of electron states [4] in DFT makes the opening of the band gap relatively small as compared to experiment [9], which in turn might limitate the theory prediction for practical application of freestanding silicene [20]. Motivated thereby, below we explore the effect of perperdicular electric fields on the bare electronic structure using DFT+DMFT within the ionic Hubbard model framework [15, 16,21].

To make progress, in Fig. 3 we display our orbitalresolved GGA results for the bare electronic structure of freestanding silicene, showing good agreement with earlier calculations [49]. It can be observed in this figure that the bare GGA DOS exhibits degenerate $3 p_{x, y}$ bands crossing the Fermi energy $\left(E_{F}=\omega=0\right)$. As seen, the $s p^{2}$ bonding-antibonding band gap splitting found in hexagonal carbon-based systems is strongly reshaped in this two-channel electron gas at normal conditions. Within this low-buckled structural phase the $p_{z}$ orbital is slightly more localized as compared to planar graphene, implying stronger electron interactions within the $\pi$-states of silicene. Importantly, our bare GGA results in Fig. 3 are consistent with earlier calculations for freestanding silicene, showing Dirac-like band dispersion and non-vanishing electronic states with $\sigma$ orbital character at low energies [49]. How this MO electronic state relevant for future valleytronics $[19,50]$ is reshaped by sizable MO electron-electron interactions, electric field and site-diagonal disorder is our focus below.

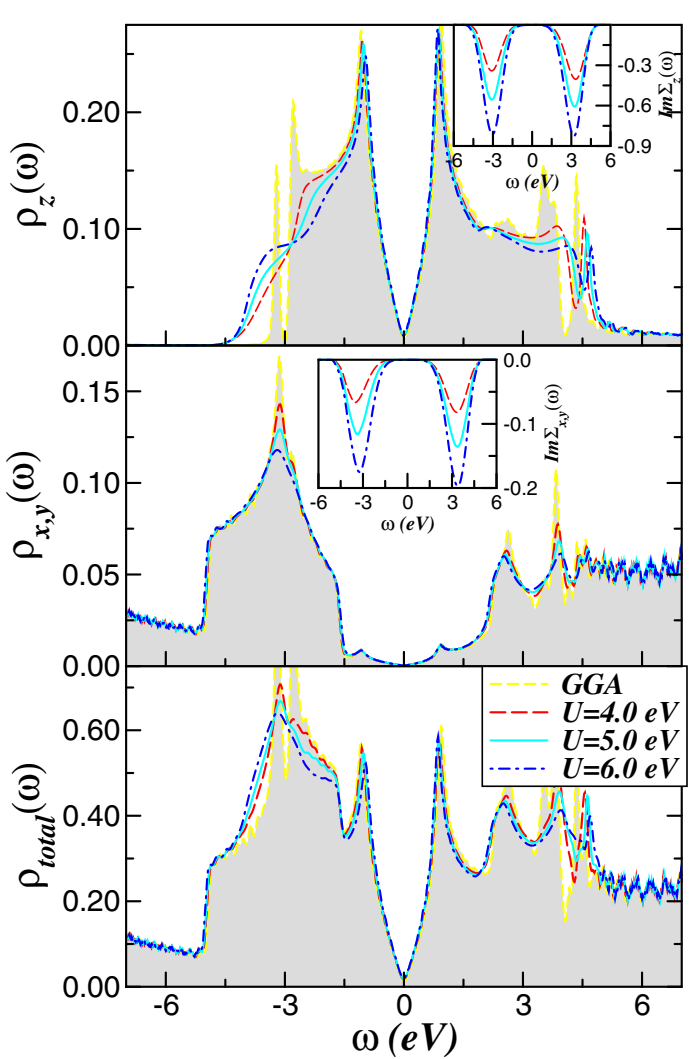

Fig. 3 GGA (dashed) and GGA + DMFT [for three different $U$ values and fixed $\left.J_{H}=0.4 \mathrm{eV}\right]$ orbital-resolved and total density-of-states (DOS) of silicene. Notice the stability of the Dirac liquid electronic state near the Fermi energy $\left(E_{F}=\omega=0\right)$ against Coulomb interaction [51] and the crossing point at $2.83 \mathrm{eV}$ binding energy induced by dynamical transfer of spectral weight. Insets show the Coulombdependence of the orbital-resolved self-energy imaginary parts

\subsection{GGA + DMFT model and results}

Within GGA, the one-electron part of the many-body Hamiltonian for silicene is $H_{0}=\sum_{\mathbf{k} a \sigma} \epsilon_{a}(\mathbf{k}) c_{\mathbf{k} a \sigma}^{\dagger} c_{\mathbf{k} a \sigma}+$ $\sum_{i a \sigma} \epsilon_{a}^{(0)} n_{i a \sigma}$, where $a=x, y, z$ labels the diagonalized $3 p$-bands, $\epsilon_{a}(\mathbf{k})$ is the one-electron band dispersion, which encodes details of the one-electron (GGA) band structure, and $\epsilon_{a}^{(0)}$ are on-site orbital energies of silicene, whose bare values are read off from the GGA spectral functions. These are relevant one-particle inputs for MO-DMFT which generates a Dirac liquid semimetal for realistic $U$ values [10] as shown below. Thus, in light of the sizable electron correlation effects in silicene-based systems [10,15, 16,27], local MO manyparticle interactions must be included to describe the onset of correlated spectral functions. These constitute the interaction term $H_{i n t}=U \sum_{i a} n_{i a \uparrow} n_{i a \downarrow}+$ $\sum_{i a \neq b} U^{\prime} n_{i a} n_{i b}-J_{H} \sum_{i a \neq b} \mathbf{S}_{i a} \cdot \mathbf{S}_{i b}$. Here, $U^{\prime} \equiv U-$ $2 J_{H}$ and $U\left(U^{\prime}\right)$ is the intra- (inter-) orbital Coulomb repulsion and $J_{H}$ is the Hund's rule coupling. The DMFT self-energy, $\Sigma_{a}(\omega)$, requires a solution of the MO 
quantum impurity problem self-consistently embedded in an effective medium [25]. Here, we use the diagrammatic MO iterated perturbation theory (MOIPT) [52] as impurity solver to DMFT. This perturbative, many-particle scheme has a proven record of describing $\mathrm{MO}$ electronic correlations in broad $p$ band systems $[18,30-33]$ and unconventional electronic structure reconstruction induced by dynamical quantum fluctuations in strongly correlated electron systems $[34,35]$. The MO-IPT scheme is computationally very efficient, with real frequency output at zero and finite temperatures, enabling the study of electronic structure reconstruction of real materials, and as shown recently it gives results for the spectral functions and self-energies in qualitatively accord with numerical exact continuos-time quantum Monte Carlo (CT-QMC) calculations $[53,54]$.

Since the fundamental problem of the Dirac liquid stability against strong Coulomb interaction is currently under debate [51], in Fig. 3 we display our GGA + DMFT results for fixed $J_{H}=0.4 \mathrm{eV}[18,30]$, showing that a weak quantum correlated scenario is applicable to silicene. Several interesting features compared to GGA are manifested in Fig. 3: a combined effect of local, MO interactions arising from $U, U^{\prime}$ and $J_{H}$ leads to spectral weight redistribution over large energy scales and the formation of an electronic structure similar to topological Kondo systems $[55,56]$, with concomitant appearance of lower- and upper Hubbard bands at high energies at the $p_{z}$ orbital sector with increasing $U$. However, the most salient features to be seen in Fig. 3 is the stability of Dirac spectrum against sizable MO electron-electron interactions and the changes in the orbital-resolved self-energy imaginary parts (see insets in Fig. 3), where the emergent Dirac liquid state is characterized by the absence of electronic excitation within the low energy window where $\operatorname{Im} \Sigma_{a}(\omega)$ vanishes.

To elucidate the role played by a perpendicular electric field $[15,16,20]$, which differentiates the on-site energies of the two sublattices of silicene, we break the sublattice symmetry by adding a scalar potential $\pm \Delta$ in the out-of-plane direction. This potential is known as ionic potential $[17,21,57]$, and the corresponding ionic Hamiltonian for silicine reads $H_{\text {ionic }}=$ $H_{0}+H_{i n t}+\sum_{\sigma}\left[\varepsilon_{A} \sum_{i \in A} n_{i z \sigma}+\varepsilon_{B} \sum_{i \in B} n_{i z \sigma}\right]$. Hence, under the influence of the external electric field the $p_{z}$ carries hop on a bipartite lattice with two sublattices $A$ and $B$ having different on-site energies $\varepsilon_{A}=-\Delta$ and $\varepsilon_{B}=+\Delta$.

In Fig. 4 we show the effect of the external electric field on the total DOS as well as on the $p_{z}$ electronic states at sites $A$ and $B$ of silicene. Consistent with earlier studies $[4,9,15,16]$, the main effect of the perpendicular field is to break the symmetry between the $A$ and $B$ sublattices and hence to open a band gap in the correlated electronic structure of silicene. As seen, as the field strength increases the on-site energy difference between the two sublattices is enhanced via charge-density-wave (CDW) ordering [58] and, consequently, the gap widens. However, few important features should be noted in our site-resolved DOS of Fig. 4.

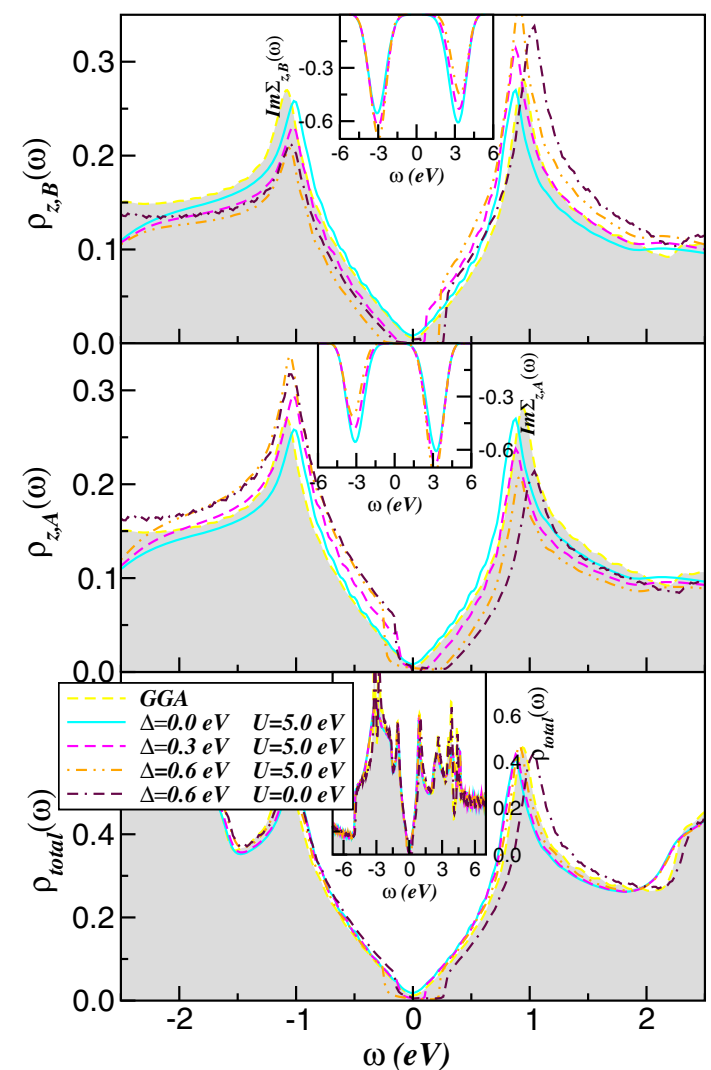

Fig. 4 Site-resolved and total GGA $+\operatorname{DMFT}(U=$ $\left.5.0 \mathrm{eV}, J_{H}=0.4 \mathrm{eV}\right)$ DOS for electric fields $(\Delta)$ applied perpendicular to the silicene hexagonal plane. Notice the fieldinduced one-particle band gap and the kink in the valence and conduction bands at sites $A$ and $B$, respectively. Insets show the weak influence of $\Delta$ in the site-resolved self-energy imaginary parts. The GGA and the $U=0.0 \mathrm{eV}$ result at large $\Delta$ are shown for comparison

Firstly, the self-energies imaginary parts remain close to that found at zero field at low energies. This implies that Dirac liquids are not only stable against strong Coulomb interactions [51] but also to CDW order. The second important feature to be seen in our results is the appearance of a kink in the valence and conduction band of $A$ and $B$ sites, respectively. Interestingly, while the $p_{z}$ DOS at site $B$ shows a clean band gap, residual electronic states appear in the $A$ site spectral function. Residual and asymmetric in-gap electronic states have been reported in Refs. [3,9], implying similar electronic structure reconstruction in spite of different electronic structure calculations. Although almost vanishing, kinks are also visible at similar energies as in Fig. 4 in the electronic spectra of Ref. [9], attesting the quality of the DFT + DMFT treatment to describe the electronic structure reconstruction of Dirac fermions under strong external electric fields $(\Delta=0.3 \mathrm{eV}$ corresponds to a perpendicular electric field of $3.0 \mathrm{~V} / \mathrm{nm}$, which is consistent with values used in Refs. $[4,8,18])$.

Let us turn our attention to the effect of sitediagonal disorder in the weakly correlated CDW elec- 


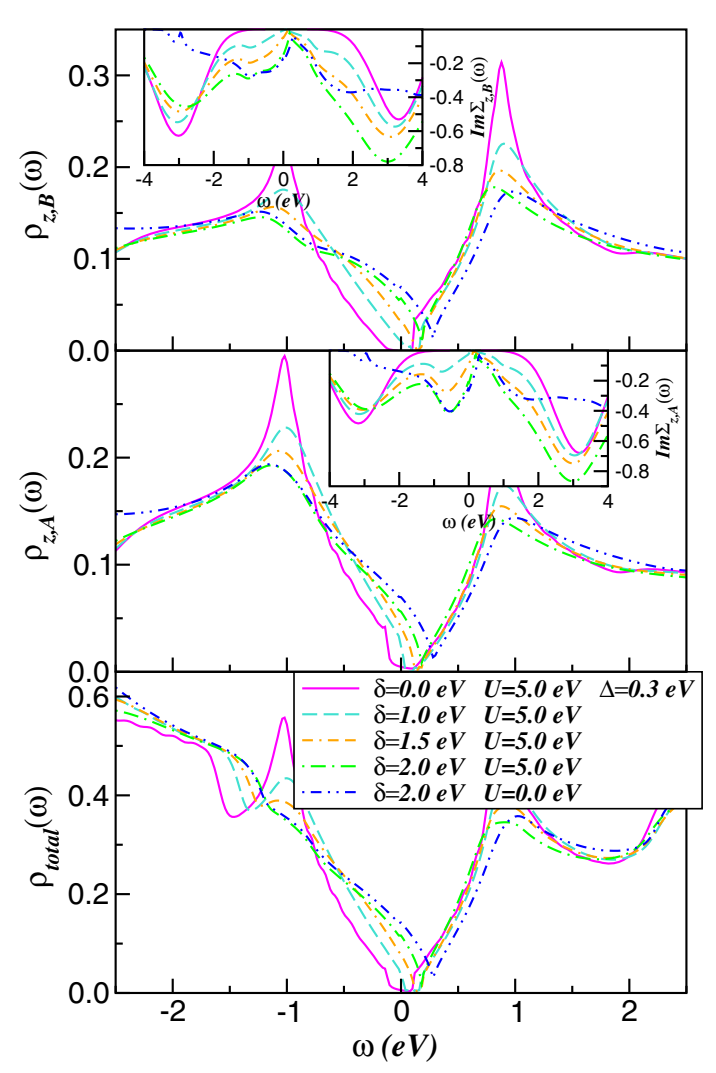

Fig. 5 Effect of site-diagonal disorder for $U=5.0 \mathrm{eV}$ and $x=0.25$ in the electronic structure of charge ordered silicene, showing strong electronic reconstruction and the emergence of marginal Dirac liquid at $\delta \geq 1.5 \mathrm{eV}$. Noteworthy is the insulator-metal crossover and the frequency dependence of the self-energy imaginary parts (insets) with increasing disorder. Notice as well the important role of disorder in the $U=0.0 \mathrm{eV}$ limit of silicene

tronic state of silicene. Here, we treat the MO problem of silicene within the DFT + DMFT(MO-IPT + CPA) approach [59], which allows for an exact treatment of binary disorder within DMFT [24]. This treatment has been already used to describe electronic phase transitions of doped Mott insulators [60,61], which were found to be in good accordance with experimental observations. Generally speaking, our scheme is an extension of that applied to the disordered Hubbard model [24], where the effect of site-diagonal disorder is modeled by incorporating a disorder term $H_{\text {dis }}=\sum_{i a \sigma} v_{i} n_{i a \sigma}$ in $H_{\text {ionic }}$ above. In the spirit of Refs. $[24,62,63]$ we restrict ourselves to a binary-alloy distribution for disorder, therefore the disorder potentials $v_{i}$ are specified by the probability distribution $P\left(v_{i}\right)=(1-x) \delta\left(v_{i}\right)+x \delta\left(v_{i}-\delta\right)$, meaning that upon incorporation of (structural or chemical) disorder a fraction $x$ of sites have an additional local potential $\delta$ for an electron hopping onto that site. In other words, the Si-3 $p$ carriers of silicene experience different local environments in the course of their hopping, and the physical object which accounts for this effect within DMFT is the coherent potential approximation (CPA) [24,64].
The combined $U+\delta$ (Mott-Anderson) problem, i.e., the situation where MO Coulomb repulsion and disorderinduced electronic reconstruction simultaneously affect the one-particle response, is treated within GGA + DMFT by a proper combination of the interaction selfenergy with the CPA one [24]. The detailed formulation of the Mott-Anderson problem has already been developed and used in the context of DFT+DMFT for real materials [59-61], so we do not repeat the equations here.

Figure 5 shows the changes in the Dirac liquid electronic structure of silicene for fixed $\Delta$ and $x=0.25$. The most salient feature to be seen is that strong electronic delocalization occurs upon the incorporation of sitediagonal disorder, suppressing the band gap induced by the external electric field. This is characterized by the appearance of low-energy electronic states near $E_{F}$. What is the origin of these low-energy features? In a disorder system, incoherent scattering between different carriers in orbital states leads to site- and orbitaldependent shifts of the $p$-bands relative to each other, and renormalized scattering rates [65] due to sizable $\delta$ cause appreciable spectral weight transfer over large energy scales, from high to low energies. This leads to a self-consistent modification of the spectral lineshape, as shown in Fig. 5. In accordance with earlier studies [24,65-68], broadening-induced quantitative changes of the effective electric field strength in the present case relative to disorder-free silicene lead to a reduced band gap lineshape, where enhanced lowenergy spectral features within the conduction band states clearly show up above $E_{F}$. Microscopically, incoherent scattering arising from site-diagonal disordered yields the emergence of reconstructed via disorder pseudogaped spectral functions with similarities akin to marginal Fermi liquids [34,35]. Noteworthy, as seen in the insets of Fig. 5, in disordered silicene the Dirac liquid behavior (with vanishing $\operatorname{Im} \Sigma_{z}$ at low energies) is extinguished by sizable local disorder effects. Thus, our incoherent metal is predicted to be the disorder counterpart of the marginal Fermi liquid found in quasifreestanding graphene [28]. More theoretical and experimental work on dirty Dirac semimetals [69] are called for to establish or refute our prediction.

To further understand the stability of the marginal Dirac liquid electronic state of disordered silicene, in Fig. 6 we display the site-resolved and total GGA + DMFT spectral functions obtained using single-zeta (SZ) and double-zeta plus polarization (DZP) basis sets [70]. This is motivated by the fact that the projected GGA DOS within the SZ basis set used to compute the correlated GGA + DMFT spectral functions above introduces a small energy shift of $0.34 \mathrm{eV}$ in the van Hove singularity at the Dirac band edge above $E_{F}$ as compared to DZP (see the lower inset of Fig. 6): we found that the precise position of the Dirac band edge in the projected GGA DOS depends on the basis set employed in the calculation, with the DZP result giving more accurate agreement between the projected DOS and the bare bandstructure (Fig. 2) of free-standing silicene. As seen in Fig. 6, the marginal Dirac liquid 


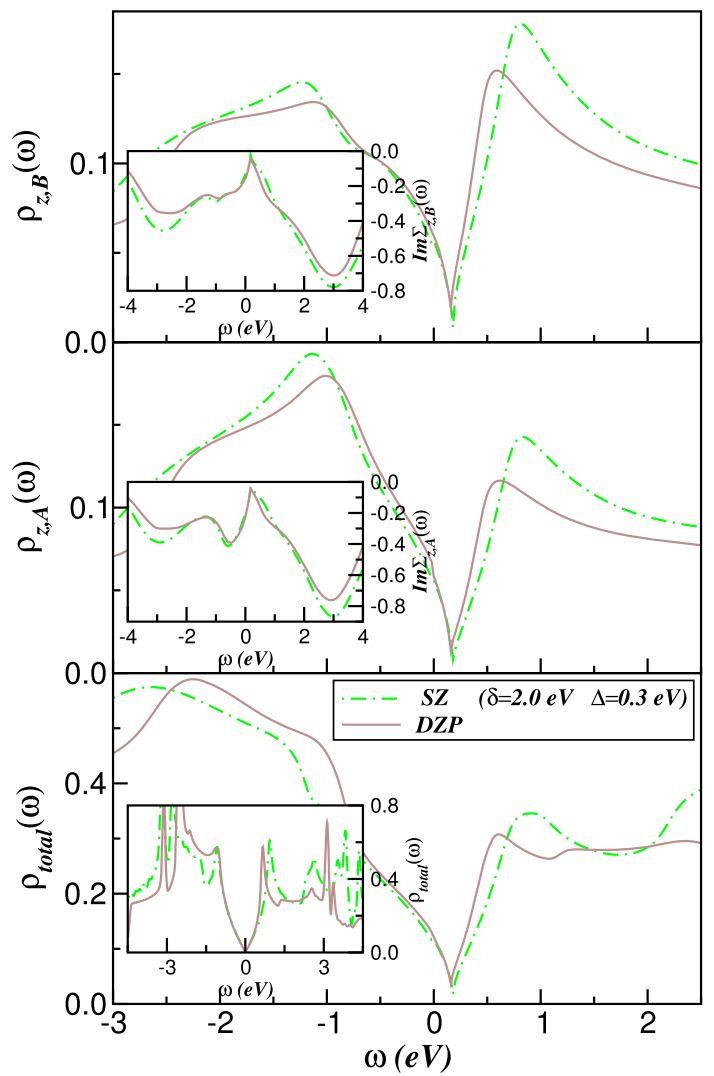

Fig. 6 Site-resolved and total GGA + DMFT spectral function obtained using two different first principles basis sets for the projected GGA DOS, showing the stability of the marginal Dirac liquid electronic state against high energy changes of the bare one-particle input to DMFT(MO-IPT + CPA). Lower inset shows the total SZ and DZP GGA DOS of free-standing silicene

electronic state is an stable fixed point, in spite the large in energy spectral weight changes in the bare GGA DOS, implying as shown below that this unconventional electronic state is intrinsically linked to the interplay between the site-diagonal disorder and the bare $V$-shaped Dirac-like electronic state.

Finally, we recall here that a fundamental important question regarding the electronic structure of silicene is whether the Dirac fermion physics, characteristic of the hexagonal lattice structure, is retained when silicene is grown on substrates. Particularly when silicene is grown on $\operatorname{Ag}(111)$ substract, where spectral features seen in the experiment of the formed superstructures $[3,8,11]$ could be intrinsic or derived from bulk and interface $\mathrm{Ag}$ states [49]. As shown in the $a b$ initio study of Ref. [49], the $p_{z}$ states (dotted lines in Fig. 7) become delocalized due to strong hybridization with the $\operatorname{Ag}(111)$ substrate, losing as a consequence its intrinsic two-dimensional honeycomb character at low energies. Furthermore, according to Ref. [49] the spatial extension of the silicene $p_{z}$ states allow them to hybridize with the Ag $s p$-states, drastically renormalizing the $\pi$ band electronic structure as compared to free-standing

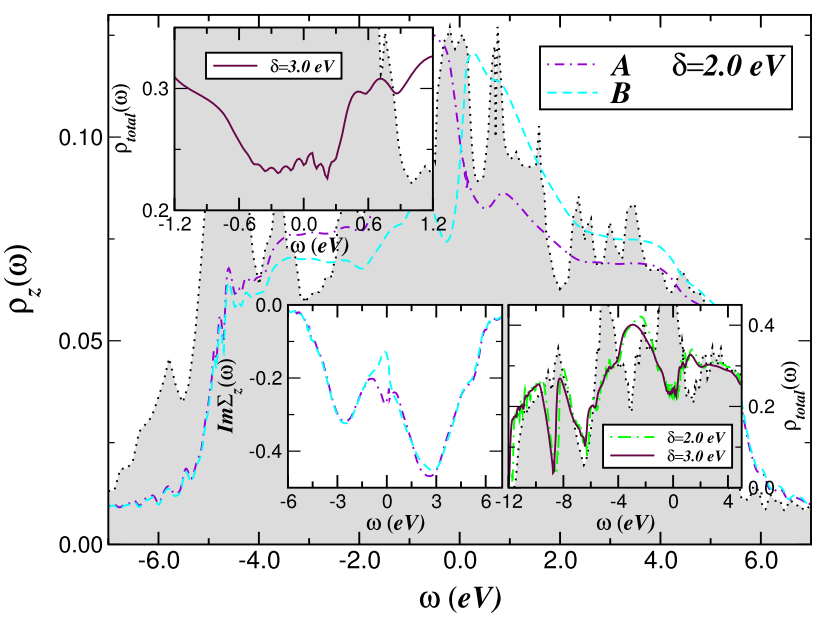

Fig. 7 Effect of combined Coulomb interaction $(U=$ $5.0 \mathrm{eV})$, electric field $(\Delta=0.3 \mathrm{eV})$ and site-diagonal disorder $(\delta)$ in the $p_{z}$ (main panel) and total (right lower panel) DOS of the $(4 \times 4)$ silicene phase. Notice in the main panel the absence of the linear Dirac band dispersion in the bare (dotted line) $p_{z}$ DOS [49]. Also noteworthy is the electronic polarization induced by the electric field (main panel) and the frequency dependence of the self-energy imaginary parts (left lower inset), showing strong deviations from the marginal Dirac liquid regime. Left upper panel display the total DOS near $E_{F}$, showing good qualitative agreement our result for $\delta=3.0 \mathrm{eV}$ and the $\mathrm{d} I / \mathrm{d} V$ spectra of Ref. [8]

silicene. Moreover, due to interorbital rehybridization a small contribution from the planar orbitals (not shown) also emerges at $E_{F}$ [49]. Thus, taken together with extant $\mathrm{d} I / \mathrm{d} V$ data from Refs. $[3,8,11]$ of silicene on $\mathrm{Ag}$ substrates revealing renormalized (strong or not) Diraclike features, seems to suggest that depending on the grown conditions the $\mathrm{Si}-\mathrm{Ag}$ hybridization can considerably modify the electronic structure of silicene, particularly the shape and energy position of the Dirac valley. Therefore, to confirm whether the marginal Dirac liquid nature of disordered silecene is preserved when the lowenergy linear band dispersion is not included as part of the bare band input to DMFT, in Fig. 7 we show the combined effect of Coulomb interaction $(U=5.0 \mathrm{eV})$, electric field $(\Delta=0.3 \mathrm{eV})$ and site-diagonal disorder $(\delta=3.0 \mathrm{eV})$ on the electronic structure reconstruction of $(4 \times 4)$ silicene, predicting the absence of marginal Dirac liquidness in this and related $(\sqrt{13} \times \sqrt{13}$ and $\sqrt{3} \times \sqrt{3})$ [49] monolayer superstructures. As seen in this figure, the self-energy imaginary parts do not follow the expected marginal behavior shown in the insets of Fig. 5, giving rise instead to an electronic spectra characteristic of strongly disordered systems treated within CPA [71] (see the main and right lower panel of Fig. 7). Surprisingly, our result for $\delta=3.0 \mathrm{eV}$ in the left upper panel of Fig. 7 is consistent with extant $\mathrm{d} I / \mathrm{d} V$ spectra of Ref. [8], showing strong particle-hole asymmetry and two-band dips followed by shoulders as in experiment, albeit at slightly different energies. This, in turn, confirms that the marginal Dirac liquid regime of free 


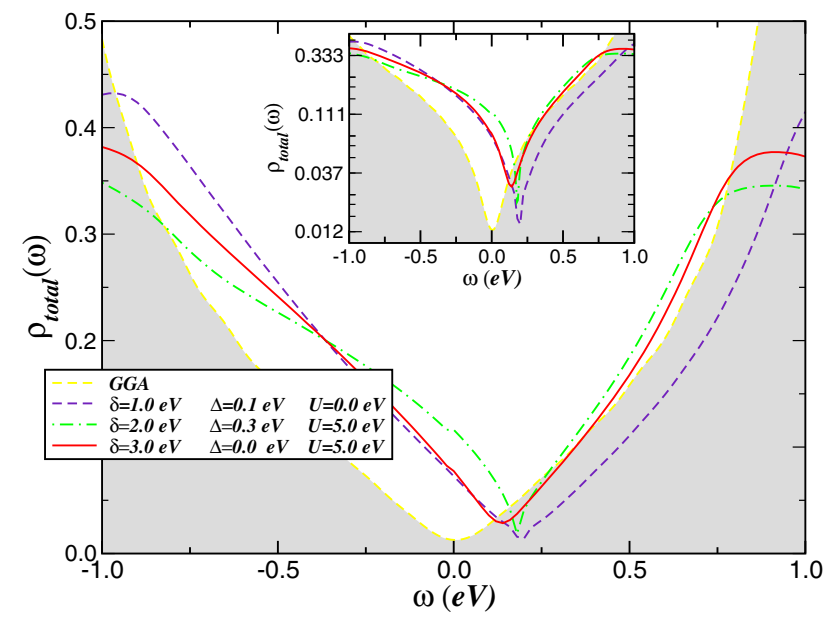

Fig. 8 Total GGA + DMFT DOS of free-standing silicene under different disorder $(\delta)$ and external electric field $(\Delta)$ conditions. The interplay between various competing effects in silicene is visible: Notice the stability of the Dirac liquid against strong disorder, showing nearly linar dispersion close to the $V$-valley minimum for $\delta=3.0 \mathrm{eV}$, and the marginal Dirac liquid DOS for $\Delta=0.3 \mathrm{eV}$. Inset shows our results in log-scale: Although at sligtly different energies, this electronic structure reconstruction is in good qualitative agreement with scanning tunneling spectroscopy data recorded in silicene on Ag substrates [3]. The GGA total DOS is shown for comparison

standing silicene is unstable against strong interaction between silicene and the metal substract [8], in contrast with $\mathrm{d} I / \mathrm{d} V$ data reported in Refs. $[3,11]$ as well as with the linear dispersion seen in angle-resolved photoelectron spectroscopy (ARPES) measurements [5], where the Dirac lineshape is preserved. However, whether the marginal Dirac liquid behavior holds true to other selforganized superstructure phases of silicene grown on $\mathrm{Ag}$ or alternative metal substracts remain to be seen in future studies.

\section{Discussion and conclusion}

The questions that we ask and answer in this work are important for understanding the electronic nature of disordered $p$-band Dirac liquids and for engineering silicene on functional subtracts for future nanotechnology devices based on field effect transistors $[9,12]$. An affirmative theoretical answer to electronic structure reconstruction of silicene is shown in Fig. 8, which is in good qualitative agreement with extant STS curves on silicene on $\mathrm{Ag}$ substract $[3,9,11]$. Our spectral functions in Fig. 8 reproduce the main STS signals, showing overall metallic character and different power law behaviour near the neutrality point, albeit at sligtly different energies for the Dirac band minima. According to our results the top atoms feel stronger electric field effects, due to tip effects in STS [72], while the botton atoms are more prompt to disorder due to their proximity with Ag substrate. Importantly, our GGA + DMFT results capture the most salient features seen in experiment. Particularly interesting is the $V$-shape form for $\delta=3.0 \mathrm{eV}$, suggesting avoided quantum criticallity as recently discussed in the context of disordered Weyl semimetals [73]. Also salient is the strange electronic behavior, characteristic of dirty Dirac semimetals near to quantum criticality [69], found at finite electric field and moderate disorder $(\delta=2.0 \mathrm{eV})$ which is in good accord with STS data. In this regime our GGA + DMFT result correctly reproduces the shoulder below the Dirac point as well as the spectral lineshape for the top atoms $[3,11]$ of silicene. Taken theory and experiment together, it is interesting that a GGA + DMFT calculation is able to access the emerging band anisotropy of dirty Dirac semimetals near to quantum criticality [69].

In summary, using GGA + DMFT we resolve the nature of the Dirac liquid regime in silicene. Good semiquantitative accord with key tunneling data in a quantum correlated picture confirms that charge carriers in silicene interact via Coulomb repulsion [10]. Our results show emergent quantum criticality of Dirac fermions in the presence of disorder and translational symmetry breaking on the honeycomb lattice. In this strange Dirac liquid regime the electronic lineshape is in good qualitative agreement with extant scanning tunneling spectroscopy data $[3,11]$, implying that the interplay between disorder and perpendicular electric field plays a major role in understanding the low energy electronic structure of silicene on Ag substrate. Our microscopic description of intrinsic multi-orbital electronic interactions and external perturbations is expected to be generally applicable to disordered $p$-band semimetals $[74,75]$ of pivotal importance.

L.C.'s work is supported by CNPq (Grant No. 304035/20173). Acknowledgment (L.C. and S.S.C.) is also made to CAPES. S.L. thanks ARCCA Cardiff for computational resources. Via S.L.'s membership of the UK's HPC Materials Chemistry Consortium, which is funded by EPSRC (No. EP/L000202), this work made use of the facilities of ARCHER, the UK's National High-Performance Computing Service, which is funded by the Office of Science and Technology through EPSRC's High End Computing Programme.

\section{Author contributions}

SSC carried out the GGA calculations. LC designed and carried out the GGA + DMFT study. The authors contributed to the scientific discussions and the preparation of the manuscript, and approved the final version of the manuscript.

Funding Open Access funding enabled and organized by Projekt DEAL. 
Data Availability Statement This manuscript has no associated data or the data will not be deposited. [Authors' comment: The datasets generated and analyzed during the current study are available from the corresponding author upon reasonable request.].

Open Access This article is licensed under a Creative Commons Attribution 4.0 International License, which permits use, sharing, adaptation, distribution and reproduction in any medium or format, as long as you give appropriate credit to the original author(s) and the source, provide a link to the Creative Commons licence, and indicate if changes were made. The images or other third party material in this article are included in the article's Creative Commons licence, unless indicated otherwise in a credit line to the material. If material is not included in the article's Creative Commons licence and your intended use is not permitted by statutory regulation or exceeds the permitted use, you will need to obtain permission directly from the copyright holder. To view a copy of this licence, visit http://creativecomm ons.org/licenses/by/4.0/.

\section{References}

1. S. Cahangirov, M. Topsakal, E. Aktr̈k, H. ahin, S. Ciraci, Phys. Rev. Lett. 102, 236804 (2009)

2. S. Lebègue, O. Eriksson, Phys. Rev. B 79, 115409 (2009)

3. D. Chiappe, C. Grazianetti, G. Tallarida, M. Fanciulli, A. Molle, Adv. Mater. 24, 5088 (2012)

4. N.D. Drummond, V. Zólyomi, V.I. Fal'ko, Phys. Rev. B 85, 075423 (2012)

5. P. Vogt, P. De Padova, C. Quaresima, J. Avila, E. Frantzeskakis, M.C. Asensio, A. Resta, B. Ealet, G.L. Lay, Phys. Rev. Lett. 108, 155501 (2012)

6. Y. Feng, D. Liu, B. Feng, X. Liu, L. Zhao, Z. Xie, Y. Liu, A. Liang, C. Hu, Y. Hu, S. He, G. Liu, J. Zhang, C. Chen, Z. Xu, L. Chen, K. Wu, Y.-T. Liu, H. Lin, Z.-Q. Huang, C.-H. Hsu, F.-C. Chuang, A. Bansil, X.J. Zhou, PNAS 113, 14656 (2016)

7. see also, R. Quhe, Y. Yuan, J. Zheng, Y. Wang, Z. Ni, J. Shi, D. Yu, J. Yang, J. Lu, Sci. Rep.4, 5476 (2014)

8. B. Feng, Z. Ding, S. Meng, Y. Yao, X. He, P. Cheng, L. Chen, K. Wu, Nano Lett. 12, 3507 (2012)

9. Z. Ni, Q. Liu, K. Tang, J. Zheng, J. Zhou, R. Qin, Z. Gao, D. Yu, J. Lu, Nano Lett. 12, 113 (2012)

10. M. Schüler, M. Rösner, T.O. Wehling, A.I. Lichtenstein, M.I. Katsnelson, Phys. Rev. Lett. 111, 036601 (2013)

11. M. Houssa, A. Dimoulas, A. Molle, J. Phys. Condens. Matter 27, 253002 (2015)

12. A. Molle, C. Grazianetti, L. Tao, D. Taneja, M.H. Alam, D. Akinwande, Chen. Soc. Rev. 47, 6370 (2018)

13. J. Guerrero-Sánchez, D.M. Munoz-Pizza, N. Takeuchi, Appl. Surf. Sci. 479, 847 (2019)

14. J. Zhuang, X. Xu, H. Feng, Z. Li, X. Wang, Y. Du, Sci. Bull. 60, 1551 (2015) (references therein)

15. L.-D. Zhang, F. Yang, Y. Yao, Sci. Rep. 5, 8203 (2015)

16. V. Julian, E. Federico, A.J. Sebastian, J. Paula, J. Magn. Magn. Mater. 524, 167598 (2021)

17. E. Adibi, S.A. Jafari, Phys. Rev. B. 93, 075122 (2016)

18. L. Craco, D. Selli, G. Seifert, S. Leoni, Phys. Rev. B 91, 205120 (2015)
19. Y. Li, H.B. Zhu, G.Q. Wang, Y.Z. Peng, J.R. Xu, Z.H. Qian, R. Bai, G.H. Zhou, C. Yesilyurt, Z.B. Siu, M.B.A. Jalil, Phys. Rev. B 97, 085427 (2018)

20. J.-A. Yan, S.-P. Gao, R. Stein, G. Coard, Phys. Rev. B 91, 245403 (2015)

21. L. Craco, P. Lombardo, R. Hayn, G.I. Japaridze, E. Müller-Hartmann, Phys. Rev. B 78, 075121 (2008)

22. V. Janiš, D. Vollhardt, Phys. Rev. B 46, 15712 (1992)

23. V. Dobrosavljević, G. Kotliar, Phys. Rev. Lett. 71, 3218 (1993)

24. M.S. Laad, L. Craco, E. Müller-Hartmann, Phys. Rev. B 64, 195114 (2001)

25. G. Kotliar, S.Y. Savrasov, K. Haule, V.S. Oudovenko, O. Parcollet, C.A. Marianetti, Rev. Mod. Phys. 78, 865 (2006)

26. S.A. Jafari, Eur. Phys. J. B 68, 537 (2009)

27. L.-D. Zhang, F. Yang, Y. Yao, Phys. Rev. B 92, 104504 (2015)

28. D.A. Siegel, C.-H. Park, C. Hwang, J. Deslippe, A.V. Fedorov, S.G. Louie, A. Lanzara, PNAS 108, 11365 (2011)

29. See also, H. Dai, J. Hou, X. Zhang, Y. Liang, T. Ma, (2020). arXiv:2009.14647 (unpublished and references therein)

30. L. Craco, M.S. Laad, S. Leoni, A.S. de Arruda, Phys. Rev. B 87, 155109 (2013)

31. L. Craco, S. Leoni, Phys. Rev. B 85, 075114 (2012)

32. L. Craco, S. Leoni, Phys. Rev. B 85, 195124 (2012)

33. L. Craco, S. Leoni, Sci. Rep. 5, 13772 (2015)

34. L. Craco, S. Leoni, Sci. Rep. 7, 46439 (2017)

35. L. Craco, Phys. Rev. B 101, 085131 (2020)

36. I.S. Tupitsyn, N.V. Prokofev, Phys. Rev. Lett. 118, 026403 (2017)

37. L. Craco, T.A. da Silva Pereira, S.R. Ferreira, S.S. Carara, S. Leoni, Phys. Rev. B 98, 035114 (2018)

38. C.M. Varma, P.B. Littlewood, S. Schmitt-Rink, E. Abrahams, A.E. Ruckenstein, Phys. Rev. Lett. 63, 1996 (1989)

39. A. Georges, G. Kotliar, W. Krauth, M.J. Rozenberg, Rev. Mod. Phys. 68, 13 (1996)

40. J. Hofmann, E. Barnes, S. Das Sarma, Phys. Rev. B 92 , 045104 (2015)

41. J.M. Soler, E. Artacho, J.D. Gale, A. Garc'ı, J. Junquera, P. Ordejón, D. Sánchez-Portal, J. Phys. Condens. Matter 14, 2745 (2002)

42. E. Artacho, E. Anglada, O. Dieguez, J.D. Gale, A. Garc'ıa, J. Junquera, M. Martin, P. Ordejón, J.M. Pruneda, D. Sánchez-Portal, J.M. Soler, J. Phys. Condens. Matter 20, 064208 (2008)

43. H.J. Monkhorst, J.D. Pack, Phys. Rev. B 13, 5188 (1976)

44. N. Troullier, J.L. Martins, Phys. Rev. B 43, 1993 (1991)

45. J.P. Perdew, K. Burke, M. Ernzerhof, Phys. Rev. Lett. 77, 3865 (1996)

46. W. Kohn, L.J. Sham, Phys. Rev. 140, A1133 (1965)

47. J. Junquera, Ó. Paz, D. Sánchez-Portal, E. Artacho, Phys. Rev. B 64, 235111 (2001)

48. J. Moreno, J.M. Soler, Phys. Rev. B 45, 3891 (1992)

49. P.M. Sheverdyaeva, S.K. Mahatha, P. Moras, L. Petaccia, G. Fratesi, G. Onida, C. Carbone, ACS Nano 11, 975 (2017) 
50. J.R. Schaibley, H. Yu, G. Clark, P. Rivera, J.S. Ross, K.L. Seyler, W. Yao, X. Xu, Nat. Rev. Mater. 1, 16055 (2016)

51. I.S. Tupitsyn, N.V. Prokofév, Phys. Rev. Lett. 118, 026403 (2017)

52. L. Craco, Phys. Rev. B 77, 125122 (2008)

53. L. Craco, S. Leoni, Phys. Rev. B 100, 121101(R) (2019)

54. L. Craco, S. Leoni, Phys. Rev. B 102, 045142 (2020)

55. S.-L. Yu, X.C. Xie, J.-X. Li, Phys. Rev. Lett. 107, $010401(2011)$

56. D.A. Pesin, L. Balents, Nat. Phys. 6, 376 (2010)

57. A. Garg, H.R. Krishnamurthy, M. Randeria, Phys. Rev. Lett. 97, 046403 (2006)

58. E. Christou, F. de Juan, F. Krüger, Phys. Rev. B 101, $155121(2020)$

59. L. Craco, Solid State Commun. 253, 14 (2017)

60. L. Craco, M.S. Laad, S. Leoni, E. Müller-Hartmann, Phys. Rev. B 70, 195116 (2004)

61. L. Craco, C.I. Ventura, A.N. Yaresko, E. MüllerHartmann, Phys. Rev. B 73, 094432 (2006)

62. K. Byczuk, M. Ulmke, D. Vollhardt, Phys. Rev. Lett. 90, 196403 (2003)

63. H. Terletska, C.E. Ekuma, C. Moore, K.-M. Tam, J. Moreno, M. Jarrell, Phys. Rev. B 90, 094208 (2014)

64. M.A. Korotin, Z.V. Pchelkina, N.A. Skorikov, E.Z. Kurmaev, V.I. Anisimov, J. Phys. Condens. Matter 26, $115501(2014)$

65. M.M. Radonjić, D. Tanasković, V. Dobrosavljević, K. Haule, Phys. Rev. B 81, 075118 (2010)
66. K. Byczuk, W. Hofstetter, D. Vollhardt, Phys. Rev. Lett. 94, 056404 (2005)

67. M.A. Gusmão, Phys. Rev. B 77, 245116 (2008)

68. R.D.B. Carvalho, M.A. Gusmão, Phys. Rev. B 87, $085122(2013)$

69. A. Szabo, B. Roy, Phys. Rev. Res. 2, 043197 (2020)

70. R.Q. Zhang, Q.Z. Zhang, M.W. Zhao, Theor. Chem. Acc. 112, 158 (2004)

71. M.S. Laad, L. Craco, J. Phys. Condens. Matter 17, 4765 (2005)

72. L. Craco, G. Cuniberti, Braz. J. Phys. 32, 293 (2002)

73. J.H. Wilson, D.A. Huse, S. Das Sarma, J.H. Pixley, Phys. Rev. B 102, 100201 (2020)

74. M. Knap, J.D. Sau, B.I. Halperin, E. Demler, Phys. Rev. Lett. 113, 186801 (2014)

75. Y. Xu, J. Chiu, L. Miao, H. He, Z. Alpichshev, A. Kapitulnik, R.R. Biswas, L.A. Wray, Nat. Commun. 8, 14081 (2017) 